\title{
Energy Management Using a Situational Awareness- Centric Ad-Hoc Network in a Home Environment
}

\author{
Tannaz Monajemi, Ardavan Rahimian, and Kamyar Mehran \\ School of Electronic Engineering and Computer Science, \\ Queen Mary University of London, London E1 4NS, UK \\ $\{$ a.rahimian, k.mehran\} @qmul.ac.uk
}

\begin{abstract}
Energy management theory and techniques for home environments are facing several technical challenges in areas including the real-time scheduling, power distribution, and automation of network of home appliances/renewables for achieving maximum energy efficiency. In this paper, situational awareness (SA) has made this crucial decision making process more efficient, by providing the valuable data about the surrounding environment. In a smart home, apart from the electrical appliances, the intelligent sensors are also consuming energy while transmitting data or when they are in idle mode. In this contribution, our focus is on implementing and analysing a situational awareness-based ad-hoc network in a home environment, in order to reduce the energy consumption, and therefore, increasing the lifetime of these networks. The presented results demonstrate the effectiveness of the proposed SA-centric method, and further confirm the energy consumption in the intended environment is decreased dramatically due to the applied schedule and limitations on the working hours of the devices. Moreover, the sensors are switched to doze mode when there is no data to exchange.
\end{abstract}

Keywords: Ad-hoc network; home energy management; situational awareness.

\section{Introduction}

The energy consumption in wireless networks is recently undergoing an intense study, and several methods and architectures are proposed to manage the energy usage in these ubiquitous networks. Wireless energy analysis, measurement, and management based on the situational awareness (SA) strategies tries to explore the elaboration of schemes for the efficient distributed computing and monitoring of the execution of plans, based on the high-performance techniques [1]. Furthermore, the SA-centric schemes are also employed for the efficient deployment of a number of strategic applications, including the cybersecurity [2-3], Internet of things (IoT) and big data [4-6], power systems and smart grid [7-14], and computer, communication, and mobile networks [15-18].

There are various questions that SA aims to answer. Some of these questions are about choosing the sensors' allocation method (i.e., dynamically or statically) in mobile ad-hoc networks (MANETs), and the way data can be potentially collected from these sensors through the specified monitoring algorithms, such as the threshold- and valuemonitoring. Other questions concerning how to achieve a certain level of SA using the information provided by monitoring methods, and how to mine the collected data using the mining algorithms incorporating the online analytical mining (OLAM) techniques, 
as well as the stream computing. The aim of this investigation is to demonstrate that a situational awareness-based system could be a very powerful and efficient solution to manage the energy allocation of an ad-hoc infrastructure within a home environment, and to extend the lifetime of batteries used in the employed wireless sensor networks (WSNs). Decision making process is a very challenging task within this platform, since the SA server has to conduct the prediction of the next optimum state of each node, as well as the most efficient path between any source and destination node. Accomplishing these intended objectives is viable using the real-time and accurate data provided by the intelligent sensors utilised as part of the deployment of the SA-centric platform.

\section{MANETs and Medium Access Methods}

In order for the defined terminals to be able to communicate within a multiple access network over a shared medium, channel access control mechanisms are required which are provided by the medium access protocol (MAC) within the infrastructure. At first, random access protocols did not check the channel availability before any transmission. After some enhancements, another random access method called carrier sense multiple access protocol (CSMA) was proposed, in which nodes sense the transmission medium before exchanging any data. In order to overcome the stumbling blocks of the previous protocols, multiple access with collision avoidance (MACA) protocol was proposed. In this method, two kinds of short-frames are used. The node that plans to transfer its data sends request to send (RTS) message, and the destination node replies by sending the clear to send (CTS) message to clarify that it can receive the data. The neighbour nodes would then recognise these messages, and would not send any data during the intended transmission over the channel. Furthermore, IEEE 802.11 MAC is also designed for a single channel, and network performance and spatial reuse of the wireless channels can take place by using the directional antennas and multiple channels. The newly proposed protocol is called multichannel MAC protocol with directional antennas (MMAC-DA) that benefits from IEEE 802.11 power saving mechanism (PSM). The nodes exchange controlling packets via announcement traffic indication message (ATIM) to choose a data channel and detect a beam direction for exchanging a traffic. This protocol results in a better throughput, packet delivery, and efficient energy consumption, as well as a proper fairness in the network. Each node has two transceivers in a way that one is used for exchanging the controlling packets, and the other is used for data transmission in various channels. This protocol enhances the spatial reuse of a wireless channel, as well as saving the energy of wireless nodes. Moreover, using the bidirectional antennas can also improve the spatial reuse of the medium channels. In order to improve the network capacity, directional network allocation vector (DNAV) is then employed instead of the NAV and by using the circular direction RTS in MAC (CDR-MAC), RTS is transmitted in a directional consecutive fashion, which helps the receiver to identify the location of the sender; then the receiver replies by sending a directional CTS (CTS-MAC) message in the exact direction of the received RTS. All the directional RTS/CTS messages are sent in the multihop directional MAC (MMAC). The sender uses the multihop RTSs in order to set up a connection to the particular destination, then they exchange CTS, data, and ACK in directional mode over a single hop. Considering the RTS/CTS messages, 
nodes can collect information about the location of their neighbours, the angle of arrival (AoA), as well as the received signal power, so that they could predict the direction and distance of the intended destination, and to form a beam toward it. The time structure of IEEE 802.11 PSM is used to divide time into beacon intervals where each beacon is divided into ATIM window and data window. There are two types of channels in this model, including a controlling channel $(\mathrm{CCH})$ and data channels $(\mathrm{DCHs})$. In previous models, free data channels were wasted since all the nodes had to be on the $\mathrm{CCH}$ during the ATIM window. In comparison, the new model allows for the nodes to employ the DCH during the ATIM window. During a high load of the network, some nodes are exchanging controlling packets over the $\mathrm{CCH}$, while others are transmitting data on the chosen DCHs, i.e. the transmission can be extended to the next ATIM window [19].

\section{Overview of Situational Awareness}

Situational awareness (SA) was first proposed during World War I by Oswald Boelke who defined it as "the importance of gaining an awareness of the enemy before the enemy gained a similar awareness, and devised methods for accomplishing this". More specifically, in an engineering and technical context, SA is defined as the perception of elements in the environment within a volume of time and space and the comprehension of their meaning and a projection of their status in the near future (i.e., Fig. 1) [1, 12].

Wireless sensor networks have been widely employed in diverse environments from battlefields to remote dangerous volcanic areas, to collect appropriate and accurate data and therefore, SA has provided real-time information for better decision making. As such, in order to tackle cyber-attacks, enterprises are benefiting from cyber situational awareness framework based on OODA (i.e., observe, orient, decide, and act) technique [3]. Automatic identification system (AIS) is also employed as the collision avoidance mechanism in the maritime situational awareness by which ships send their presence, identification, and location [21]. Deploying wide-area situational awareness (WASA) for power grids has provided valuable data that is used for monitoring, archiving, and envisioning the state of the dynamic system via connected sensors [7]. In spite of the key role of the monitoring and tracking capabilities in emerging business opportunities, IoT has not been adopted as expected, due to the specific requirements of the systems. In 1999, Tim Bass proposed the cyberspace situational awareness (CSA) concept [5]. In addition, SA is used in WSN that can be formed in a hierarchical framework in which the cluster heads are responsible for a number of tasks including the routing, connection to other clusters and neighbours, and monitoring and administration of the nodes [6]. Dealing with the missing data, by using the information available in an environment where the SA is deployed, interpolation is the best way in order to estimate the missing data for a short period of time, while historic load features are more beneficial when dealing with lost data in a longer time [9]. The other usage of information collected in the SA-centric system is to present the connections of the components of the network using a weighted directed graph, in which the measurements of incidental values are calculated by analysing the mobility of an observer which is moving stochastically from one component to the other, based on the deployed ambient intelligence scenario [18]. 


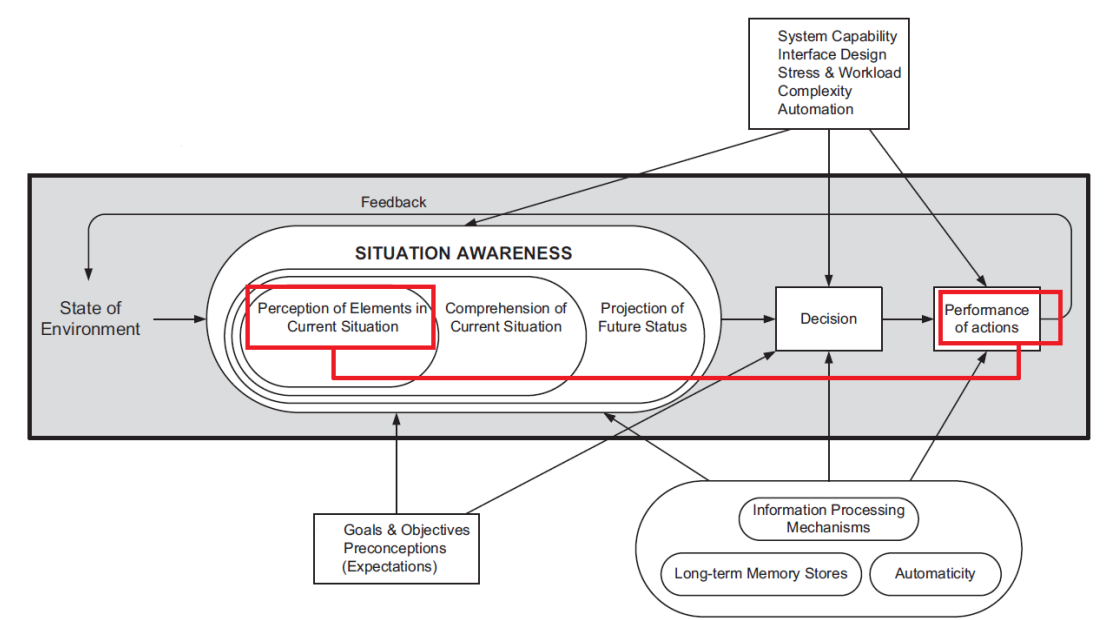

Fig. 1. The generalised model of application-specific situational awareness ${ }^{1}$, highlighting the primary domain of the focused investigation for the energy management layered framework.

\subsection{WSNs for SA-Centric Systems}

Wireless sensor networks for situational awareness (WSN-SA) systems incorporate six primary sections, including the sensing component, advanced ad-hoc communication, through-the-debris communication, radio frequency (RF) band for SA-centric systems, dual-operational mode, and ultra-low power wake-up radio (WOR) technology. These components are not completely independent of each other. In a sensing device, sensors that are utilised in the SA-centric systems might be different from the ones utilised in WSNs. An attention has been dedicated to next generation of sensors, called intelligent sensors with the ability to perform in ultra-low power mode and further recognising the environmental events in a fraction of a second. The other important component is the dual-operation mode of WSNs. The common protocols developed for the WSNs mainly require nodes to be woken-up several times per second which makes the nodes work in the full-operation (FO) mode, and is exactly the same as the original SA systems which is not energy-efficient. The other functionality mode is called a limited-operation (LO), in which a network would be virtually activated and deactivated several times a day, and nodes would exchange their states to the main SA server in order to examine the intrinsic health of the network. Switching between the FO and LO modes is conducted by commands via a base station (BS). When a disaster is recognised by the local nodes, the efficient environmental deployed sensors are forced to switch to the FO mode.

\subsection{Network Design and Performance Analysis}

As mentioned earlier, the focus of this investigation has been mainly on the design and performance evaluation of a situational awareness-based ad-hoc network employed for

${ }^{1}$ This picture is adopted from [12], but customised for our proposed SA-centric framework for energy management in a home environment. 
the purpose of reducing the energy consumption in a home environment, which results in the potential increase in the lifetime of these networks. In a smart home environment, the overall energy consumption, denoted as $E_{t}$, can be calculated as a combination of the energy used by the home appliances (i.e., $E_{a}$ ), and the energy that sensors consume while they are awake sensing the environment and conducting the data transmission, as well as the consumption rate while in their idle or doze modes (i.e., $E_{s}$ ). Therefore, the total energy consumption in the intended environment can be expressed as follows:

$$
\stackrel{\text { Total }}{\longrightarrow} E_{t}=E_{a}+E_{s}
$$

The SA servers play the most critical roles in the process, since they are considered as the primary decision makers. The most crucial information is provided to these servers by the intelligent sensors so that they can make the most appropriate decisions in a realtime manner. Once the decision is made, the SA server would provide the sensors with the optimum time for the data exchange, best route to the required destination, and list of the neighbours and their location, as well as the angle that each node has to conduct the beamforming during the data exchange process. In addition, to find the pattern of the energy consumption, the SA server would not require the utilised intelligent sensors to be awake continuously, but at certain times of the day that could reduce the energy consumption by the sensors. The SA server would allocate a schedule for each sensor node in order to sense the surrounding environment in the FO mode and after that when no packets are exchanged, nodes would be forced to switch to the LO mode. Hence, by switching to the LO mode, the network would have deactivated and activated several times a day. The sensor nodes consume various amount of energy according to their corresponding status. The intelligent sensor consumes $1.65-\mathrm{W}, 1.4-\mathrm{W}$, and $1.15-\mathrm{W}$, when functioning in the transmission, reception, and idle modes, respectively $[19,20]$. The first simulated scenario shows the energy efficiency enhancement in the SA-centric ad-hoc network versus the conventional ad-hoc network in which nodes that have no packets to exchange would switch to doze mode, in contrast with the idle mode in IEEE 802.11 while facing the same situation. The total number of sensors are 100, data packet size is 512 bytes, packet arrival rate increases over time up to 100 packets per second, and network energy efficiency is calculated using equation (2). The upper- and lowerbound network throughputs are considered as $80 \%$ and $20 \%$, respectively. In each of these conditions, due to the network topology, various proportion of the nodes might contribute in routing the packets from a source to a destination. The probability of nodes contributing in a routing process varies from 0.3 to 0.6 , and each node provides various results for the overall network energy efficiency, as presented in Figs. 2 (a) and (b).

$$
\text { Network Energy Efficiency }=\frac{\text { Total Energy Consumption }}{\text { Number of Delivered Packets }}
$$

According to the presented simulations, implementing the situational awareness-centric ad-hoc network has reduced the network's energy consumption, based on the employed sensors being forced by the SA server to switch into the doze mode, when they do not have any packets to exchange. The intended optimum network performance in both conditions, considering the upper- and lower-bounds, are achieved when the nodes with the cooperation probability of 0.3 are routing the packets. In all the scenarios, energy 
consumption in the SA-based network is less than the conventional ad-hoc network. As also mentioned earlier, in the newly proposed protocol (MMAC-DA), time is divided into the beacon intervals and at the start of each beacon there is an announcement traffic indication message (ATIM) window; each beacon is divided into an ATIM window and a data window. The controlling messages are transmitted via the $\mathrm{CCHs}$, and the data is transferred by the DCHs. The protocol benefits from the PSM, and has further enhanced it so that more simultaneous packets could be sent over the multiple channel resources. This process is accomplished by using a controlling channel as a data channel during a data window. In the presented simulated scenario, the SA server schedules the beacon interval, ATIM window, and data window. At the start of the interval, the source node $(S)$ checks the status of the destination node $(D)$, and if available, it would send the ATIM message to the destination node in order to perform the channel negotiation, and to provide the information about the transmission power, as well as to detect the beam direction. By analysing this message, node $D$ would understand the beam direction to node $S$, and would select a data channel. Also, node $D$ sends the ACK message to node $S$ clarifying the chosen data channel and beam direction. Receiving this message, node $S$ confirms the selected channel and beam direction by sending the ATIM reservation (A-RES) message. Once this stage is completed, both nodes $S$ and $D$ would transmit a directional reservation message (DRES) to each other, as the last stage prior to the initiation of the data exchange process. Neighbour nodes would further overhear these handshaking messaged between $S$ and $D$, and would then update their CUL.

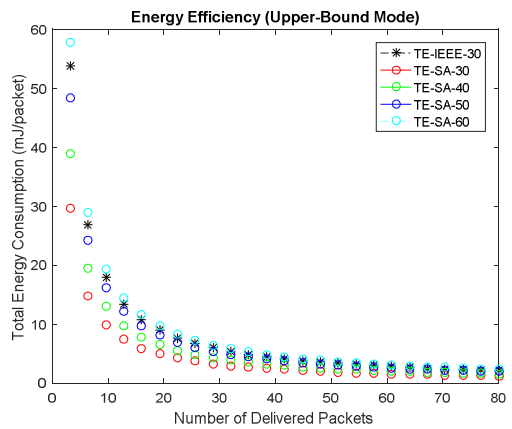

(a)

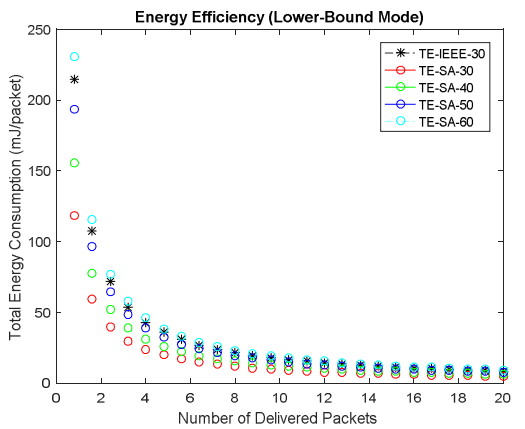

(b)

Fig. 2. Energy-efficiency plots for the upper- and lower-bounds of the proposed SA-centric ad-hoc system, based on the total energy consumption and number of delivered packets.

In the data window, $S$ and $D$ would switch to the selected data channel to exchange data and the other nodes would switch to the doze mode in which they consume less energy (i.e., 0.045-W), rather than the defined idle mode. In addition, when the traffic load of the network increases, controlling channels can be used as data channels during the data window, which further results in more successful packets being transferred from the source to the intended destination. This result in the substantial increase in the overall efficient system performance in the SA-based ad-hoc network. Figs. 3 (a) and (b) presents thoroughly, the simulated scenario, in which the total simulation time is 10 seconds, and the packet size and number of nodes are 512 bytes and 100, respectively. Also, the total number of channels are 3 . The probability of packets being successfully delivered in each channel considering both the lower- and upper-bounds are 0.55 and 
0.85. The network throughput is calculated based on the following equation (3), which is increased significantly using more channels for the data exchange operation.

$$
\text { Network Throughput }=\frac{\text { Packet Size } \times \text { Number of Delivered Packets }}{\text { Total Simulation Time }}
$$

The SA server indicates the beacon interval, and then at the start of the ATIM window, the source and destination would initiate the handshaking process using the controlling channel. At the start of the next beacon interval, the SA server would force the utilised sensors to employ this controlling channel in addition to the predefined data channels. This process would result in the increase of the channel's spatial reuse in the intended environment. Consequently, more packets are being transmitted simultaneously, based on the soft-real time (SRT) network framework (i.e., the ability to withstand a certain amount of delay) [22]. Moreover, for the appliances to reduce the energy consumption, they need to use energy only in specific hours of the day. As a case in point, when no one is in the house, the cooling system, television, and lights should be switched off. Detecting this event (i.e., nobody being present in the house), the sensor would activate its cluster head $(\mathrm{CH})$; i.e., the SA node in the hibernation mode; and then would report this waste of energy in that specific surrounding area. Afterwards, the SA node would wake up its $\mathrm{CH}$ (i.e., the SA server) via transmitting the beacons specified for this task, for conducting the data transmission. The server would process the data and if required, it would ask for more information from that area of the house, to make decisions.

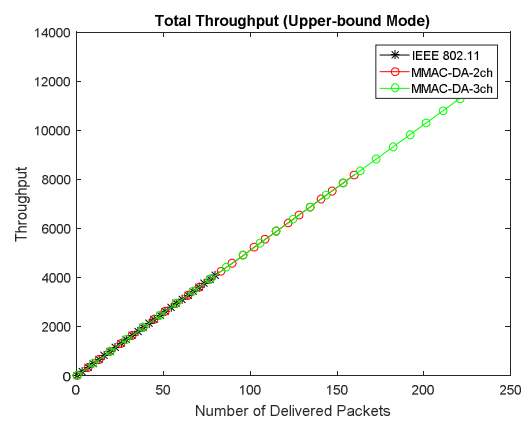

(a)

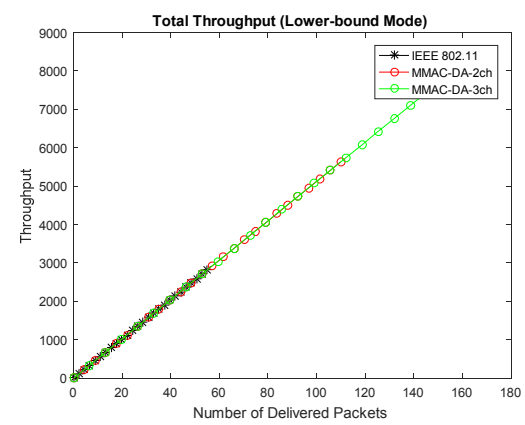

(b)

Fig. 3. Network throughput plots for the upper- and lower-bounds of the proposed SA-centric ad-hoc system, based on the throughput and number of delivered packets.

Hence, by collecting the adequate amount of information, the applied SA server would decide which appliances to switch off. This task is conducted by the supervisory SA agent in the various areas of the house that would give the command to the intelligent sensors to switch off these appliances, which results in an efficient energy consumption. Furthermore, there are a number of factors that should also be taken into account while deploying the physical (PHY) layer elements, for the efficient energy transmission in the network. As a case in point, the path loss elements should be considered (i.e., link budget planning) while the intended scenarios are deployed in a real environment [23]. To demonstrate a functionality assessment of the proposed SA framework, a scenario is considered and analysed, which consists of three utilised intelligent sensors in a home 
environment controlling the operation of a television, a fan, and a fluorescent light bulb in a room. The power consumption of the utilised devices is $0.06,0.05$, and $0.01 \mathrm{kWh}$, respectively, and the average electricity unit price is 15.5 pence per $\mathrm{kWh}$. Sensors with power supply of $5-\mathrm{V}$ and current of $14-\mathrm{mA}$, consume $7.5 \times 10^{-7} \mathrm{kWh}$ in the idle mode. Hence, the total energy consumption of all electrical devices within an hour would be $0.120225 \mathrm{kWh}$. Assuming the energy could be saved by this mechanism for 3 hours per day; as part of the considered SA-centric scenario; at the end of the year, 131.646375 $\mathrm{kWh}$ and $21 \mathrm{GBP}$ could be saved. Therefore, by connecting other electrical appliances in a home environment to the proposed SA-centric ad-hoc network, the overall power consumption over a year, would be significantly decreased.

\section{Conclusion}

We have proposed the thorough design and performance analysis of an ad-hoc network based on the situational awareness framework. The proposed architecture has reduced the energy consumption in a home environment, for the purpose of implementing a regulated and efficient home energy management framework. Moreover, the presented SA platform improves the functionality of wireless networks by providing valuable information about the appropriate way of distributing resources, and also predicts the feasible risks, resulting in a more efficient energy consumption. This investigation can be extended into the case of network modelling and performance analysis based on the SA-based game-theoretical security enhancement, and adaptive dynamic programming (ADP)-based system realisation, within a real-time large-scale architecture.

\section{References}

1. Howard, N., Cambria, E.: Intention awareness: improving upon situation awareness in humancentric environments. Human-centric Computing and Information Sciences. 3, 1-17 (2013)

2. Franke, U., Brynielsson, J.: Cyber situational awareness - A systematic review of the literature. Computers \& Security. 46, 18-31 (2014)

3. Lenders, V., Tanner, A., Blarer, A.: Gaining an Edge in Cyberspace with Advanced Situational Awareness. IEEE Security \& Privacy. 13, 65-74 (2015)

4. Wu, J., Ota, K., Dong, M., Li, J., Wang, H.: Big Data Analysis-based Security Situational Awareness for Smart Grid. IEEE Transactions on Big Data. (2016)

5. Gendreau, A.A.: Situation Awareness Measurement Enhanced for Efficient Monitoring in the Internet of Things. In: IEEE Region 10 Symposium (TENSYMP), pp. 82-85. (2015)

6. Gendreau, A.A., Barrios, R.M.: Hierarchical-Based Measurement of Situation Awareness in the Internet of Things. In: International Conference on Wireless Networks (ICWN). (2014)

7. Basu, C., Padmanaban, M., Guillon, S., Cauchon, L., De Montigny, M., Kamwa, I.: Situational awareness for the electrical power grid. IBM Journal of Research and Development. 60, 10:1-10:11 (2016)

8. He, X., Qiu, R.C., Ai, Q., Chu, L., Xu, X., Ling, Z.: Designing for Situation Awareness of Future Power Grids: An Indicator System Based on Linear Eigenvalue Statistics of Large Random Matrices. IEEE Access. 4, 3557-3568 (2016) 
9. Peppanen, J., Reno, M.J., Thakkar, M., Grijalva, S., Harley, R.G.: Leveraging AMI Data for Distribution System Model Calibration and Situational Awareness. IEEE Transactions on Smart Grid. 6, 2050-2059 (2015)

10. Giri, J., Parashar, M., Trehern, J., Madani, V.: The Situation Room: Control Center Analytics for Enhanced Situational Awareness. IEEE Power and Energy Magazine. 10, $24-39$ (2012)

11. Chen, H., Zhang, L., Mo, J., Martin, K.E.: Synchrophasor-based real-time state estimation and situational awareness system for power system operation. Journal of Modern Power Systems and Clean Energy. 4, 370-382 (2016)

12. Panteli, M., Kirschen, D.S.: Situation awareness in power systems: Theory, challenges and applications. Electric Power Systems Research. 122, 140-151 (2015)

13. Dahal, N., Abuomar, O., King, R., Madani, V.: Event stream processing for improved situational awareness in the smart grid. Expert Systems with Applications. 42, 6853-6863 (2015)

14. Alcaraz, C., Lopez, J.: WASAM: A dynamic wide-area situational awareness model for critical domains in Smart Grids. Future Generation Computer Systems. 30, 146-154 (2014)

15. Wang, C., Lin, H., Zhang, R., Jiang, H.: SEND: A Situation-Aware Emergency Navigation Algorithm with Sensor Networks. IEEE Transactions on Mobile Computing. (2016)

16. Eiza, M.H., Owens, T., Ni, Q., Shi, Q.: Situation-Aware QoS Routing Algorithm for Vehicular Ad Hoc Networks. IEEE Transactions on Vehicular Technology. 64, 5520-5535 (2015)

17. Rolim, C.O., Rossetto, A.G., Leithardt, V.R.Q., Borges, G.A., Geyer, C.F.R., dos Santos, T.F.M., Souza, A.M.: Situation awareness and computational intelligence in opportunistic networks to support the data transmission of urban sensing applications. Computer Networks. (2016)

18. Roy, S., Dhal, R.: Situational Awareness for Dynamical Network Processes Using Incidental Measurements. IEEE Journal of Selected Topics in Signal Processing. 9, 304-316 (2015)

19. Dang, D.N.M., Nguyen, V.D., Tra Le, H., Hong, C.S., Choe, J.: An efficient multi-channel MAC protocol for wireless ad hoc networks. Ad Hoc Networks. 44, 46-57 (2016)

20. Silva, A.R., Liu, M., Moghaddam, M.: WSN-SA: Design Foundations for Situational Awareness Systems Based on Sensor Networks. In: IEEE Global Humanitarian Technology Conference (GHTC), pp. 179-184. (2013)

21. Papi, F., Tarchi, D., Vespe, M., Oliveri, F., Borghese, F., Aulicino, G., Vollero, A.: Radiolocation and tracking of automatic identification system signals for maritime situational awareness. IET Radar, Sonar \& Navigation. 9, 568-580 (2015)

22. Mehran, F., Rahimian, A.: Physical Layer Performance Enhancement for Femtocell SISO/MISO Soft Real-Time Wireless Communication Systems Employing Serial Concatenation of Quadratic Interleaved Codes. In: 20 th Iranian Conference on Electrical Engineering (ICEE), pp. 1188-1193. (2012)

23. Rahimian, A., Mehran, F.: RF Link Budget Analysis in Urban Propagation Microcell Environment for Mobile Radio Communication Systems Link Planning. In: International Conference on Wireless Communications and Signal Processing (WCSP), pp. 1-5. (2011) 